\title{
Adjuvant vaginal brachytherapy as a part of management in early endometrial cancer
}

\author{
Sylwia Kellas-Ślęczka, MD, PhD, Piotr Wojcieszek, MD, Brygida Białas, MD, PhD \\ Brachytherapy Department, Maria Skłodowska-Curie Memorial Cancer Center and Institute of Oncology. Gliwice, Poland
}

\begin{abstract}
Endometrial cancer is the most frequent cancer of female genital tract. Metro- and menorrhagia or postmenopausal bleeding results in its early presentation. It allows radical treatment. However, controversies remain on surgery coverage or adjuvant therapies in early endometrial women cancer. Optimal management should minimize intervention instead of aggressive approach, as showed by recent studies. There is a role for brachytherapy as an adjuvant irradiation. Crucial publications including PORTEC-1, GOG 99, MRC ASTEC, ASTEC/EN.5, PORTEC-2 or Italian lymphadenectomy trial are discussed. Moreover, there is attention paid on adjuvant vaginal brachytherapy analyses for the past fifteen years.
\end{abstract}

Key words: brachytherapy, endometrial cancer, HDR, LDR, vaginal vault, vaginal cylinder.

\section{Purpose}

Endometrial cancer is mostly diagnosed at the early stage. In spite of low value of population screening, postmenopausal bleedings, meno- and metrorhaggia let to detect it early. Most women have favorable prognosis after treatment, although there are some histopathologic high risk features. These are grade 3 tumor, $>50 \%$ of myometrial invasion, lymphovascular space invasion (LVSI), non-endometrioid histology (e.g. serous, clear cell, undifferentiated, small cell, anaplastic) and cervical stromal involvement [1].

Type 1 and 2 phenotypes of endometrial cancer are distinguished. Type 1 or endometrioid carcinoma is associated with hyperoestrogenism and endometrial hyperplasia. It occurs predominantly in pre- and perimenopausal women. It is associated with good prognosis. Type 2 or nonendometrioid cancer is non-estrogen dependent. It is more aggressive and with poor prognosis. It develops usually from atrophic endometrium in older women. Type 2 cancers occur in about $10 \%$ of cases, but are responsible for $50 \%$ of failures. There is a number of estrogen-dependent type 1 cancers. Type 2 cancer widespread is constant, but overall survival has decreased significantly [2-4].

\section{Surgery}

Early endometrial cancer treatment should be based on surgery. Adjuvant therapy should be given, if needed. It includes radiotherapy, brachytherapy or chemotherapy [1,5].

Standard surgery should consist of total hysterectomy and bilateral salpingo-oophorectomy (TAH + BSO). Pelvic lymphadenectomy was evaluated in two prospective ran- domized trials. MRC ASTEC contained two arms. Standard surgery $(\mathrm{TAH}+\mathrm{BSO}$, peritoneal washings and palpation of para-aortic lymph nodes) had 704 patients. It was compared to standard surgery plus lymphadenectomy in 704 patients. There was no significant differences in the 5 -year overall survivall between these two arms $(81 \%$ for standard surgery versus $80 \%$ for lymphadenectomy arm; $\mathrm{HR}=1.16, p=0.31$ ). The 5-year recurrence-free survival was in favor of standard surgery $(79 \%$ vs. $73 \%$; HR $=1.35$, $p=0.017$ ). Adjustment of differences between these two arms resulted with no statistical significance in overall survival and recurrence-free survival, either. The limitation of this study was the fact that the lymphadenectomy specified in the protocol was not comprehensive and did not include all of pelvic and para-aortic nodes. An Italian prospective randomized trial analyzed patients with early stage of endometrial cancer. Women were randomized to systematic pelvic lymphadenectomy (264 patients) or no lymph nodes removal (250 patients). Nodal involvement was diagnosed significantly more often in lymphadenectomy arm (13.3\% vs. $3.2 \%, p<0.001)$. However, there was no improvement of progression-free survival $(81 \%$ vs. $81.7 \%, p=0.68)$ and overall survival $(85.9 \%$ vs. $90 \%, p=0.50)$. In addition, women who had not lymphadenectomy suffered from less complications (81 patients vs. 34 patients, $p=0.001)[6,7]$. On the other hand, there are studies which showed survival benefit from lymphadenectomy. There is retrospective analysis of 671 patients with endometrial cancer treated in Japan from 1986 to 2004 . Standard surgery was performed with systematic pelvic lymphadenectomy (325 patients) or pelvic and para-aortic lymphadenectomy (346 patients). If intermediate
Address for correspondence: Sylwia Kellas-Ślęczka, MD, PhD, Brachytherapy Department, Maria Skłodowska-Curie Memorial Cancer Center and Institute of Oncology, Gliwice Branch, 15 Wybrzeże Armii Krajowej Str., 44-101 Gliwice, Poland, phone: +48 32278 92 53, @ e-mail: kellass@wp.pl
Received: 30.11 .2012

Accepted: 08.12.2012

Published: 23.12.2012 
or high risk of relapse was reported after surgery adjuvant treatment was offered. Overall survival was significantly longer in the pelvic and para-aortic lymphadenectomy group than in the pelvic lymphadenectomy group $(\mathrm{HR}=0.53$, $p=0.0005$ ). It was also observed in 407 patients at intermediate or high risk of recurrence. Overall survival was not related to lymphadenectomy type in low-risk patients. Multivariate analysis of prognostic factors was performed. Death risk reduction was observed among women with intermediate and high risk of relapse treated with pelvic and para-aortic lymphadenectomy ( $\mathrm{HR}=0.44, p<0.0001)$. Adjuvant therapy also improved overall survival in the patients from these groups $(\mathrm{HR}=0.48, p=0.0049)$ [8].

Preoperative imaging seems to be very helpful. It can provide the extension plan of lymphadenectomy before the surgery. There are efforts to initiate sentinel lymph node biopsy as a part of surgical staging. Cervical injection of patent blue and filtered $99 \mathrm{mTc}$-SC is shown as feasible and adequate method in endometrial cancer patients. Tumor diameter could be evaluated intraoperatively with frozen section of uterus to predict nodal involvement [9-11].

\section{Adjuvant external beam radiotherapy}

Surgery influences the choice of adjuvant treatment. External beam radiotherapy (EBRT) is less likely prescribed to women after lymphadenectomy. This effect is the strongest amongst women from intermediate risk group. It can be associated with results of clinical trials on use of adjuvant irradiation in endometrial cancer management [12]

One of the early findings on EBRT was published in 1980. 540 women with stage I endometrial cancer entered a prospective trial on postoperative EBRT. Radium vaginal brachytherapy (VBT) was given to all women after surgery. They were randomized into two groups, EBRT versus observation. Although significant reduction of vaginal and pelvic relapses was observed in EBRT group, there was no benefit on overall survival. Women with grade 3 tumors and invasion of more than $50 \%$ of myometrial thickness were more likely to benefit from EBRT. Danish Endometrial Cancer Group studied adjuvant radiotherapy in all endometrial cancer patients from 1986 to 1988 . Women with stage I endometrial cancer, grade 1 and 2 tumours and with the most superficial myometrial invasion had surgery alone. Excellent results, such as $96 \% 5$-year overall survival or $7 \%$ relapse rate in this group, confirmed no need of postoperative EBRT for these women. Moreover, 15 of 17 vaginal recurrences were salvaged with median observation time of 61 months $[13,14]$.

Multicenter prospective randomized trial was conducted in Netherlands from 1990 to 1997. It was later called PORTEC-1. It searched, whether adjuvant pelvic EBRT improves recurrence rates and overall survival in stage I endometrial cancer women. Nineteen from twenty Dutch oncology centers took part in this trial. 715 women were randomized after $\mathrm{TAH}+\mathrm{BSO}$ without routine lymphadenectomy. Main eligibility criteria included: proven endometrial cancer, any age, postoperative FIGO stage I with: grade 1 tumor and deep myometrial invasion $(>50 \%)$, grade 2 and any invasion or grade 3 with superficial invasion
$(<50 \%)$. There were two arms, pelvic EBRT versus no additional treatment. Adjuvant EBRT was planned on whole pelvis. Total dose of 46 Gy was delivered in 23 fractions in 5 days per week. AP-PA, 3 fields or box techniques were allowed. 5-year locoregional control was significantly improved in EBRT arm. It was $4 \%$ against $14 \%(p<0.001)$. Highest incidence of relapses was in vagina $(74 \%)$. The 5 -year overall survival was $81 \%$ in EBRT arm versus $85 \%$ in control arm $(p=0.31)$. Moreover, the survival after relapse was better in control arm $(p=0.02)$. It was probably due to better feasibility to salvage treatment. Multivariate analysis revealed that women older than 60 years had high risk of locoregional recurrence $(\mathrm{HR}=3.2, p=0.003)$ and cancerrelated death $(\mathrm{HR}=3.1, p=0.02)$. Also, deep myometrial invasion and grade 3 histology were associated with higher incidence of relapse, but not statisticaly significant (both $p=0.11$ ). Grade 3 histology was linked with cancer-related deaths $(\mathrm{HR}=4.9, p=0.0008)$. An update of PORTEC-1 was published recently with long-term outcomes. Locoregional relapses after 15 years were 5.8\% after adjuvant EBRT against $15.5 \%$ in no additional treatment arm $(p<0.001)$. Overall survival after 15 years was $52 \%$ in EBRT arm versus $60 \%$ in control group $(p=0.14)$. Quality of life was decreased in women after adjuvant EBRT. Significantly higher levels of urinary symptoms (urgency, need to be close to toilet, incontinence) and bowel symptoms (fecal urgency and leakage, diarhea and bowel-related limitations of daily activity) were observed in patients after pelvis irradiation $[15,16]$.

The Gynaecological Oncology Group designed GOG 99 trial. Postoperative pelvic radiotherapy was compared to no additional treatment in intermediate-risk endometrial cancer. It included 448 women with myometrial invasion, any grade of endometrial cancer and no evidence of nodal metastases (FIGO IB-IIB). It was based on GOG 33 surgical staging protocol. Surgical inclusion criteria was at least total abdominal hysterectomy, bilateral salpingo-oophorectomy with selective bilateral PL and PAL. Removal of any of enlarged or suspicious nodes was permitted. Laparoscopy was not allowed. EBRT group was planned to $50.4 \mathrm{~Gy}$ in 28 fractions. AP-PA or four-field technique was allowed to cover the whole pelvis. EBRT was initiated in 8 weeks after surgery. Relapse risk reduction by $58 \%$ was observed in EBRT arm (90\% CI, $p=0.007)$. Although women after EBRT had better survival rates at 48 months ( $92 \%$ vs. $86 \%$ ), it was not statisticaly significant. Furthermore, irradiated women had significant increase in side effects frequency and severity (hematologic, gastrointestinal, genitourinary and cutaneous, $p<0.001$ ) [17].

Adjuvant EBRT has been evaluated in joined MRC ASTEC and NCIC CTG EN.5 analysis. Women with intermediate and high risk of relapse entered these trials after surgery. Surgical staging based on lymphadenectomy or peritoneal cytology was not required. Patients were randomized into observation arm and EBRT arm. EBRT had to be initiated in 6-8 weeks (in EN.5 no later than 12 weeks) after surgery. Whole pelvis was treated to $40-46 \mathrm{~Gy}$ in $20-$ 25 fractions (EN.5 45 Gy in $25 \mathrm{fx}$ ) 5 times per week. Brachytherapy prescription was based on department policy. ASTEC patients were allowed to have irradiated upper third 
of vagina. HDR BT of $8 \mathrm{~Gy}$ in two fractions in 3-7 days, specified at $0.5 \mathrm{~cm}$ from the applicator surface or $15 \mathrm{~Gy}$ of LDR were recommended. EN.5 patients had BT according to local standards. 5 -year overall survival was $84 \%$ in both arms. Slight reduction of local relapse incidence was observed in EBRT arm, but with no improvement in overall survival or DFS. Besides, less early and late side-effects were observed in control arm (27\% vs. $57 \%$ in EBRT arm). Due to these results, authors did not suggest adjuvant EBRT as a treatment of choice in early endometrial cancer, even when there is an intermediate and high risk of failure [18].

\section{Adjuvant vaginal brachytherapy}

PORTEC-1, GOG-99 and ASTEC/EN.5 trials showed that use of pelvic irradiation improves locoregional control, however it caused unacceptable increase in toxicity. Several small, mostly retrospective analyses were published on vaginal brachytherapy (VBT). Results showed its feasibility and much lower toxicity among early endometrial cancer patients [19]. Low dose rate (LDR) radium/cesium vaginal brachytherapy was analyzed in low-risk stage one patients after $\mathrm{TAH}+\mathrm{BSO} .303$ women with grade 1 and 2 tumor, less than half myometrial invasion and nonmalignant peritoneal cytology had adjuvant VBT. Total dose of 30 Gy was given with vaginal cylinder (2.0-3.5 cm diameters). Dose was specified to $0.5 \mathrm{~cm}$ from applicator surface. Procedure was done 2-3 weeks after surgery. 30-year disease-free survival was $96.7 \%$ in this group. 15-year cancer-related survival was $100 \%$. No vaginal relapses were observed [20].

Adjuvant high dose rate (HDR) VBT was analysed in a retrospective study to find patterns of relapses and toxicity. 122 women with intermediate (78 patients) and high (44 patients) risk endometrial cancer were treated with adjuvant VBT. Three fractions of 7 Gy specified on surface of applicator was given within six weeks from TAH + BSO. Fraction dose specified $0.5 \mathrm{~cm}$ from the applicator surface ranged from $4.6 \mathrm{~Gy}$ for $3.5 \mathrm{~cm}$ in diameter cylinder to $4.9 \mathrm{~Gy}$ for $2.5 \mathrm{~cm}$ in diameter cylinder. Proximal two-thirds of vagina were irradiated. 5-year relapse-free survival was $94 \%$ for intermediate-risk group and $74 \%$ for high risk patients. Although twelve recurrences were observed, seven patients had pelvic recurrence and only two had vaginal relapse. Toxicities were observed only in grade 1 . No late effects were reported [21].

High-risk of failure stage I endometrial cancer patients were analyzed after adjuvant VBT. 124 women with stage $\mathrm{IB}$, grade 3 or stage IC endometrial cancer were treated with $\mathrm{TAH}+\mathrm{BSO}$ and node sampling. All of them had node-negative disease. Adjuvant VBT started at 3 to 18 weeks after surgery (median of 6 weeks). Proximal half or two-thirds of vagina was irradiated. Vaginal cylinders ranged from 2.0$3.5 \mathrm{~cm}$. Total dose of $21 \mathrm{~Gy}$ in three fractions was prescribed to $0.5 \mathrm{~cm}$ from the applicator surface. VBT was given over four weeks. No pelvic or vaginal relapse was observed after median follow-up time of 30 months. There were three abdominal failures. The estimated 5 -year overall survivall was $93 \%$. The 5 -year disease-free survival was $87 \%$. Bowel and urinary toxicities were not observed above grade 2 . Two patients delivered vaginal stenosis (77 years old and 84 years old) [22].
Another retrospective study evaluated adjuvant VBT in stage IB and IC endometrial cancer patients. 102 women after surgery were qualified to VBT. There were patients after total hysterectomy with BSO (48 laparoscopic-assisted; 51 abdominal approach). Three women had trasvaginal hysterectomy alone. 18 patients had total pelvic lymphadenectomy. 46 patients had lymph-node sampling. Adjuvant VBT was delivered with largest fitting vaginal cylinder (2.5-4.0 cm in diameter). Total dose of $15 \mathrm{~Gy}$ in three weekly fractions was given. Dose was specified to $0.5 \mathrm{~cm}$ from the applicator surface. It included vaginal apex and $5 \mathrm{~cm}$ of vaginal vault. The 5 -year overall survival was $84 \%$. The 5-year disease-free survival was $93 \%$. There was no statistical difference between stage IB and IC patients. Only one vaginal relapse was observed [23].

Adjuvant VBT alone was also analysed in stage II endometrial cancer patients. 15 women had TAH + BSO with surgical staging. Node-negative endometrial cancer was confirmed in pathologic report. HDR VBT was given in 8 days to total dose of $16.8 \mathrm{~Gy}$ in three fractions. 5.6 Gy fraction dose was specified to $0.5 \mathrm{~cm}$ from the applicator surface. It covered vaginal apex and $4 \mathrm{~cm}$ of vaginal vault. Largest possible applicator $(2.5-4.0 \mathrm{~cm})$ was used. There were no relapses or deaths observed in this group. Median observation time was 34 months. No adverse effects were reported [24].

Adjuvant HDR VBT was analysed retrospectively in 50 women with stage I-II endometrial cancer. There were no stage IC patients with grade 3 tumors. TAH + BSO with selective pelvic and para-aortic nodes dissection was performed. Adjuvant VBT was administered 6-8 weeks after surgery. Vaginal apex was marked with radio-opaque clip. Total dose of 30 Gy in 6 twice-a-week fractions was prescribed to $0.5 \mathrm{~cm}$ from applicator surface. The largest possible vaginal cylinder was chosen. In all, but one patient $4 \mathrm{~cm}$ was irradiated. The $100 \%$ isodose was optimized to cover vaginal vault on the chosen applicator length to vaginal apex. The 4 -year overall survivall was $97 \%$. The 2 -year relapse rate was $2 \%$. No toxicities above grade 1 were observed [25].

382 women with stage IB-IIB endometrial cancer were evaluated in Memorial Sloan-Kettering Cancer Center analysis. They underwent either TAH + BSO (313 patients) or laparoscopic-assisted vaginal hysterectomy with BSO (69 patients). Surgical staging was performed in 78 women. All of 382 patients had adjuvant HDR VBT. The total dose of $21 \mathrm{~Gy}$ was given in three fractions with two-week intervals. Vaginal cylinder diameter ranged from 2 to $3 \mathrm{~cm}$. Fraction dose of 7 Gy was specified to $0.5 \mathrm{~cm}$ from the applicator surface. If the vaginal cylinder size was less than $2.6 \mathrm{~cm}$, dose per fraction was decreased to 6 Gy. Proximal half or twothirds of vaginal vault was irradiated. Patients with grade 3 tumors had treated whole vaginal vault. The 5-year vaginal/pelvic control was $95 \%$. Age over 60 years, grade 3 tumor and LVI significantly increased relapse incidence. The 5 -year overall survival was $93 \%$. Poorer outcome was observed in women older then 60 years and with LVI. Less than $1 \%$ of patients had toxicity grade 3 or worse [26].

Adjuvant VBT was compared to adjuvant EBRT in non randomized series of 78 patients with stage I-II endometrial cancer. TAH + BSO with pelvic or pelvic/para-aortic lymphadenectomy was performed in all women. 42 patient received adjuvant VBT. Total dose of $21 \mathrm{~Gy}$ in three fractions 
was prescribed for HDR and 65 Gy for LDR. HDR VBT was given in three $7 \mathrm{~Gy}$ fractions. The dose was delivered with vaginal cylinders $(2.5-3.0 \mathrm{~cm})$ and specified to $0.5 \mathrm{~cm}$ from applicator surface. LDR patients were irradiated with two ovoids $(2.0-2.5 \mathrm{~cm})$, dose was specified to the vaginal surface. One insertion was done. EBRT patients received 45-50.4 Gy on whole pelvis with AP-PA or box techniques. EBRT was combined with VBT (dose was not stated). The 5-year overall survival and disease-free survival for both groups was $86 \%$ and $89 \%$, respectively. There were no differences between adjuvant VBT and EBRT. High-intermediate patients had 5-year disease-free survival of $94 \%$ for VBT group versus $80 \%$ in EBRT group ( $p=0.75$ ). Two patients had grade 3 rectal toxicity in EBRT group. Mild fibrosis and shortening of vaginal vault was observed in three patients after adjuvant EBRT with VBT [27].

High-risk endometrial cancer patients were analysed after adjuvant VBT. 87 women underwent $\mathrm{TAH}+\mathrm{BSO}$ and nodal assesment. $46 \%$ of them had nodal dissection. 75 patients had adjuvant HDR VBT. Most of them received total dose of 21 Gy in three 7 Gy fractions. One received total dose of $15 \mathrm{~Gy}$ in three $5 \mathrm{~Gy}$ fractions. One patient missed final fraction of $7 \mathrm{~Gy}$ and received total dose of $14 \mathrm{~Gy}$ in two fractions. Dose was prescribed to proximal half or two-thirds of vaginal vault. It was specifed to $0.5 \mathrm{~cm}$ from applicator surface. Vaginal cylinders were used $(2.0-3.5 \mathrm{~cm}$ in diameter). Twelve patients had adjuvant LDR VBT. Total dose of $30 \mathrm{~Gy}$, specified to $0.5 \mathrm{~cm}$ from applicator surface, was administered in one insertion. Three locoregional relapses were observed at median follow-up time of 52 months (4-157 months). All patients were treated with HDR VBT. Salvage treatment was performed successfully [28].

Despite good outcome and tolerance of VBT there was still need to establish optimal management of high-intermediate endometrial cancer patients. PORTEC-2 trial was conducted to compare adjuvant VBT and adjuvant EBRT in this group of women. Surgery criteria were the same as in the PORTEC-1 trial. Women were eligible if: older than 60 years with stage IB, grade 3 endometrial cancer or stage IC with grade 1 and 2 tumors, and any age with stage IIA (without grade 3 tumors with $>50 \%$ myometrial invasion). There were 214 women in EBRT arm and 213 in VBT arm. EBRT arm patients had whole pelvis radiotherapy to total dose of 46 Gy in 23 fractions, 5 days a week. Three-di- mensional conformal or multiple field techniques were allowed. Individual shielding in all fields was provided. VBT arm patients had vaginal cylinder placed in vaginal vault. Proximal half of it was covered with dose, specified to 0.5 $\mathrm{cm}$ from the surface of applicator. Total dose of $21 \mathrm{~Gy}$ in three weekly fractions was delivered with HDR units. Total dose of $30 \mathrm{~Gy}(50-70 \mathrm{cGy} / \mathrm{h})$ was given with LDR. Total dose of $28 \mathrm{~Gy}(100 \mathrm{cGy} / \mathrm{h})$ was prescribed for medium dose rate (MDR) units. LDR and MDR were planned for one session. The estimated 5-year vaginal relapse rates were 1.8\% after VBT and $1.6 \%$ after EBRT ( $p=0.74)$. There was no significanct diferrence in 5-year locoregional relapse $(p=0.17)$, however there was significantly more pelvic failures in VBT $\operatorname{arm}(3.8 \%$ vs. $0.5 \%, p=0.02)$. The estimated 5 -year overall survival was $84.8 \%$ in VBT arm and $79.6 \%$ in EBRT arm $(p=0.57)$. The 5 -year disease-free survival was $82.7 \%$ in VBT arm and $78.1 \%$ in EBRT arm $(p=0.74)$. Gastrointestinal early toxicity was higher in EBRT arm patients (53.8\% vs. $12.6 \%$, $p<0.05$ ). Vaginal mucosal atrophy (grade 1 and 2) was significantly more often in VBT arm. Diarrhoea was more frequent in EBRT arm. It was decreasing after end of irradiation, but after five years it was still significantly at higher levels than in VBT arm. Fecal leakage was increased in EBRT patients after 6 months $(11 \%$ vs. $3 \%, p<0.001)$. Its incidence remained stable during follow-up. The largest difference was observed when patients were asked if bowel symptoms limited their daily activity (EBRT arm $23 \%$ vs. VBT arm $7 \%$, $p<0.001)[29,30]$.

\section{Summary}

Optimal management of early endometrial cancer patients remains unclear. Despiting controversies with lymphadenectomy coverage, the best adjuvant therapy should be chosen. Adjuvant EBRT is efficient against pelvic failures, but early and late toxicity rates are unacceptable. Adjuvant VBT seems to be promising as a very good tool to prevent vaginal failures. Moreover, its use in stage I-II endometrial cancer patients is still growing, but target volumes in vaginal vault and fractionation schedules should be standardized (Table 1). Addition of irradiation do not improve overall survival, which is probably linked with distant failures. There are attempts to find optimal systemic regimen. First an Italian and Japanese publications showed no benefit from

Table 1. Adjuvant HDR VBT characteristics in presented publications

\begin{tabular}{|c|c|c|c|c|c|}
\hline & Total dose & Dose per fraction & Irradiated length* & Overall treatment time & Time from surgery \\
\hline Weiss et al. [21] & $21 \mathrm{~Gy}$ & $7 \mathrm{~Gy}$ & $2 / 3$ & - & 6 weeks \\
\hline Chadha et al. [22] & $21 \mathrm{~Gy}$ & 7 Gy & $1 / 2$ or $2 / 3$ & 4 weeks & 6 weeks $^{\dagger}$ \\
\hline Anderson et al. [23] & $15 \mathrm{~Gy}$ & 5 Gy & $5 \mathrm{~cm}$ & 3 weeks & - \\
\hline Rittenberg et al. [24] & $16.8 \mathrm{~Gy}$ & $5.6 \mathrm{~Gy}$ & $4 \mathrm{~cm}$ & 8 days & - \\
\hline Jolly et al. [25] & $30 \mathrm{~Gy}$ & 5 Gy & $4 \mathrm{~cm}$ & 3 weeks & 6-8 weeks \\
\hline Alektiar et al. [26] & $21 / 18 \mathrm{~Gy}^{\ddagger}$ & $7 / 6 \mathrm{~Gy}^{\ddagger}$ & $1 / 2$ or $2 / 3$ & 6 weeks & - \\
\hline Lin et al. [27] & $21 \mathrm{~Gy}$ & 7 Gy & - & - & - \\
\hline Mckloskey et al. [28] & $21 \mathrm{~Gy}$ & $7 \mathrm{~Gy}$ & - & - & - \\
\hline Nout et al. [30] & $21 \mathrm{~Gy}$ & $7 \mathrm{~Gy}$ & $1 / 2$ & 3 weeks & 6 weeks \\
\hline
\end{tabular}

${ }^{*}$ Vaginal vault length from apex; ${ }^{\dagger}$ median time, ranged from 3-18 weeks; ${ }^{*}$ prescribed dose was decreased if vaginal cylinder $<2.6 \mathrm{~cm}$ was used 
introduction of sequential cisplatin in comparison to adjuvant EBRT. In joined NSGO-EN-9501/ILIADE-III randomized pooled analysis adjuvant EBRT was compared to adjuvant chemoradiation. Progression-free survival was improved in combined modalities arm $(\mathrm{HR}=0.63, p=0.009)$. There was no benefit in overall survival, however, the trend was observed in favor of chemoradiation $(\mathrm{HR}=0.69$, $p=0.07$ ). There are still ongoing trials (GOG 249, GOG 258, PORTEC-3, Danish/EORTC) and we have to wait for the results [1,31-33].

It seems there is no need for adjuvant irradiation in stage IA, grade 1 or 2 patients. This group should also contain a patient with single failure risk factor. Adjuvant VBT should be prefered over pelvic EBRT in patients with at least 2 failure risk factors (age $>60$ years, $>50 \%$ of myometrial invasion, grade 3 , serous or clear cell tumor, LVSI). It is caused by excellent local control rates and very good treatment tolerance. Patients with more than 2 failure risk factors, stages II and III, should be considered as candidates for prospective clinical trials with chemotherapy or chemoradiation.

\section{References}

1. Amant F, Mirza MR, Creutzberg CL. Cancer of the corpus uteri. Int J Gynaecol Obstet 2012; 119 Suppl 2: S110-117.

2. Bokhman JV. Two pathogenetic types of endometrial carcinoma. Gynecol Oncol 1983; 15: 10-17.

3. Evans T, Sany O, Pearmain P et al. Differential trends in the rising incidence of endometrial cancer by type: data from a UK population-based registry from 1994 to 2006. Br J Cancer 2011; 104: 1505-1510.

4. Gottwald L, Pluta P, Piekarski J et al. Long-term survival of endometrial cancer patients. Arch Med Sci 2010; 6: 937-944.

5. Press JZ, Gotlieb WH. Controversies in the treatment of early stage endometrial carcinoma. Obstet Gynecol Int 2012; 5: 784790.

6. Kitchener H, Swart AM, Qian Q et al. Efficacy of systematic pelvic lymphadenectomy in endometrial cancer (MRC ASTEC trial): a randomized study. Lancet 2009; 373: 125-136.

7. Benedetti Panici P, Basile S, Maneschi F et al. Systematic pelvic lymphadenectomy vs. no lymphadenectomy in early-stage endometrial carcinoma: randomized clinical trial. J Natl Cancer Inst 2008; 100: 1707-1716.

8. Todo Y, Kato H, Kaneuchi M et al. Survival effect of para-aortic lymphadenectomy in endometrial cancer (SEPAL study) a retrospective cohort analysis. Lancet 2010; 375: 1165-1172.

9. Ugaki H, Kimura T, Miyatake T et al. Intraoperative frozen section assessment of myometrial invasion and histology of endometrial cancer using the revised FIGO staging system. Int J Gynecol Cancer 2011; 21: 1180-1184.

10. Cade TJ, Quinn MA, McNally OM et al. Predictive value of magnetic resonance imaging in assessing myometrial invasion in endometrial cancer: is radiological staging sufficient for planning conservative treatment? Int J Gynecol Cancer 2010, 20: 1166-1169.

11. How J, Lau S, Press J et al. Accuracy of sentinel lymph node detection following intra-operative cervical injection for endometrial cancer: A prospective study. Gynecol Oncol 2012; 127: 332-337.

12. Sharma C, Deutsch I, Lewin SN et al. Lymphadenectomy influences the utilization of adjuvant radiation treatment for endometrial cancer. Am J Obstet Gynecol 2011; 205: 562 e1-9.

13. Poulsen HK, Jacobsen M, Bertelsen K et al. Patients with early stages of endometrial cancer should be spared adjuvant radiotherapy. Danish Endometrial Cancer Group. Ugeskr Laeger 1997; 159: 3403-3407.
14. Aalders J, Abeler V, Kolstad P et al. Postoperative external irradiation and prognostic parameters in stage I endometrial carcinoma: clinical and histopathologic study of 540 patients. Obstet Gynecol 1980; 56: 419-427.

15. Nout RA, van de Poll-Franse LV, Lybeert ML et al. Long-term outcome and quality of life of patients with endometrial carcinoma treated with or without pelvic radiotherapy in the post operative radiation therapy in endometrial carcinoma 1 (PORTEC-1) trial. J Clin Oncol 2011; 29: 1692-1700.

16. Creutzberg CL, van Putten WL, Koper PC et al. Surgery and postoperative radiotherapy versus surgery alone for patients with stage-1 endometrial carcinoma: multicentre randomized trial. PORTEC Study Group. Post Operative Radiation Therapy in Endometrial Carcinoma. Lancet 2000; 355: 1404-1411.

17. Keys HM, Roberts JA, Brunetto VL et al. A phase III trial of surgery with or without adjunctive external pelvic radiation therapy in intermediate risk endometrial adenocarcinoma: a Gynecologic Oncology Group study. Gynecol Oncol 2004; 92: 744-751.

18. Blake P, Swart AM, Orton J et al. Adjuvant external beam radiotherapy in the treatment of endometrial cancer (MRC ASTEC and NCIC CTG EN.5 randomized trials): pooled trial results, systematic review, and meta-analysis. Lancet 2009; 373: 137-146.

19. Donnelly ED, Rakhra S, Helenowski I et al. Dosimetry and toxicity outcomes in postoperative high-dose-rate intracavitary brachytherapy for endometrial carcinoma. J Contemp Brachyther 2012; 4: 135-140.

20. Eltabbakh GH, Piver MS, Hempling RE et al. Excellent longterm survival and absence of vaginal recurrences in 332 patients with low-risk stage I endometrial adenocarcinoma treated with hysterectomy and vaginal brachytherapy without formal staging lymph node sampling: report of a prospective trial. Int J Radiat Oncol Biol Phys 1997; 38: 373-380.

21. Weiss E, Hirnle $\mathrm{P}$, Arnold-Bofinger $\mathrm{H}$ et al. Adjuvant vaginal high-dose-rate afterloading alone in endometrial carcinoma: patterns of relapse and side effects following low-dose therapy. Gynecol Oncol 1998; 71: 72-76.

22. Chadha M, Nanavati PJ, Liu P et al. Patterns of failure in endometrial carcinoma stage IB grade 3 and IC patients treated with postoperative vaginal vault brachytherapy. Gynecol Oncol 1999; 75: 103-107.

23. Anderson JM, Stea B, Hallum AV et al. High-dose-rate postoperative vaginal cuff irradiation alone for stage IB and IC endometrial cancer. Int J Radiat Oncol Biol Phys 2000; 46: 417-425.

24. Rittenberg PV, Lotocki RJ, Heywood MS et al. Stage II endometrial carcinoma: limiting post-operative radiotherapy to the vaginal vault in node-negative tumors. Gynecol Oncol 2005; 98: 434-438.

25. Jolly S, Vargas C, Kumar T et al. Vaginal brachytherapy alone: an alternative to adjuvant whole pelvis radiation for early stage endometrial cancer. Gynecol Oncol 2005; 97: 887-892.

26. Alektiar KM, Venkatraman E, Chi DS et al. Intravaginal brachytherapy alone for intermediate-risk endometrial cancer. Int J Radiat Oncol Biol Phys 2005; 62: 111-117.

27. Lin LL, Mutch DG, Rader JS et al. External radiotherapy versus vaginal brachytherapy for patients with intermediate risk endometrial cancer. Gynecol Oncol 2007; 106: 215-220.

28. McCloskey SA, Tchabo NE, Malhotra HK et al. Adjuvant vaginal brachytherapy alone for high risk localized endometrial cancer as defined by the three major randomized trials of adjuvant pelvic radiation. Gynecol Oncol 2010; 116: 404-407.

29. Nout RA, Putter H, Jürgenliemk-Schulz IM et al. Five-year quality of life of endometrial cancer patients treated in the randomised Post Operative Radiation Therapy in Endometrial Cancer (PORTEC-2) trial and comparison with norm data. Eur J Cancer 2012; 48: 1638-1648. 
30. Nout RA, Smit VT, Putter H et al. Vaginal brachytherapy versus pelvic external beam radiotherapy for patients with endometrial cancer of high-intermediate risk (PORTEC-2): an open-label, non-inferiority, randomized trial. Lancet 2010; 375: 816-823.

31. Hogberg T, Signorelli M, de Oliveira CF et al. Sequential adjuvant chemotherapy and radiotherapy in endometrial cancer results from two randomized studies. Eur J Cancer 2010; 46: 2422-2431.

32. Maggi R, Lissoni A, Spina F et al. Adjuvant chemotherapy vs radiotherapy in high-risk endometrial carcinoma: results of a randomized trial. Br J Cancer 2006; 95: 266-271.

33. Susumu N, Sagae S, Udagawa Y et al. Randomized phase III trial of pelvic radiotherapy versus cisplatin-based combined chemotherapy in patients with intermediate- and high-risk endometrial cancer: a Japanese Gynecologic Oncology Group study. Gynecol Oncol 2008; 108: 226-233. 